\title{
Author Index - Abstracts
}

Abel,P.W. 26,60,61 Aitken, V. 8 Alborch,E. 1,2 Allen, J.C. 3 Altura, B.M. 65 Altura, B.T. 65 Awan,N.A. 38

Baer, R.W. 19 Barja, F. 45 Beijer, H.J.M. 4 Berne, R.M. 73 Bevan, J.A. 5,74 Bevan, R.D. 6 Birkenhäger, W.H. 14 Bisetti, M.-L. 7 Bogaert, M.G. 9 Bohr, D.F. 72 Boullin, D.J. 8 Bowers, R. 51 Brutsaert, D.L. 57 Bucher, B. 62 Buylaert, W.A. 9

Cambridge, D. 10 Castro-Tavares, J. 11 Chapnick,B. 20 Charbon, G.A. 4 Chevülard,C. 75 Clemens, M.G. 12 Clough,D.P. 13,44 Coen,E.P. 70 Collis, M.G. 13,70 Cotterrell, D. 29 Couliboeuf, H. 62

Daniel,E.E. 32 DeMey,J. 15,16,27 DeRidder,W. 15 Dobbs,R.J. 48 Driessens, M.F. 17

DuBoulay, G.H. 8

Ehrlich,W. 19

Feigen, L. 20 Feniuk,W. 21 Ferrari, A. 36 Flaim,S.F. 22,77 Flatman, J.A. 41 Florence, V.M. 5 Forrester, T. 12 Fredholm, B.B. 54 Furchgott, R.F. 23

Gabella,G. 66 Gerthoffer, W.T. 3 Gootman,P.M. 65 Gordon, J.L. 64 Gorman, M.W. 56 Greenberg, S. 24 Greengrass, P.M. 10 Gregorini, L. 36 Gross, F. 30 Gurtner, G.H. 63 Haddy,F. 44 Hatton,R. 13 Heistad, D.D. 25 Hermsmeyer, K. 26,60,61 Hillman, J. 35 Hjemdahl,P. 54 Holzmann, S. 31 Hotta,K. 76 Huggel,H. 7,45 Humphrey, P.P.A. 21 Huot,S. 44 Hutchings, A. 64 Ingram, D. 26 Ingram, M.J. 26

Janczewska, H. 27 Janssen, P.A.J. 67 Janssens,W.J. 28,70 Jarman, D.A. 8 Jenden, D.J. 18 Johansson, B. 66

Kadowitz,P. 20 Karim,F. 29 Kato,H. 42 Keddie,J.R. 13 Kelsey,C.R. 48 Khayyal,M. 30 Roller, A. 49 Kovách, A.G.B. 49 Kreye,V.A.W. 30 Kukovetz, W.R. 31 Kwan,C.Y. 32 Laurent, P. 62 Leeuw,P.W. de 14 Leonetti, G. 36 Ligeti, L. 49 Ljung, B. 33 Lockette, W.E. 72 Lugnier, C. 34 Lundvall, J. 35

Maas,A.H.J. 4 Macho, P. 69 Majewski, H. 55 Mancia, G. 36 Marcus, M.L. 25 Martin, G. 1 Maruyama, M. 37 Mason,D.T. 38 Matsuki,N. 60,61 McGrath, J.C. 39 McMurty,I.F. 71 Mekata, F. 40 Mulvany, M.J. 41

Nakayama, K. 42 Nedergaard, O.A. 43

Pamnani, M. 44 Peristiány, J.G. 7,45 Piper Duckies, S. 18 Pittman,R.N. 46 Pöch, G. 31

Powis, D.A. 47

172

Author Index

Rand,M.J. 55 Reeves, J.T. 71 Robinson, B.F. 48 Rubányi, G. 49 Rubio,R. 73 Saiag, B. 75 Saverymuttu,S.H. 48 Scheftel, J.M. 50 Schneider, W. 53 Seidel,C.L. 51 Siegel,G. 53 Shepherd, A.P. 52 Shibata,S. 37 Smith, D.J. 3 Sollevi, A. 54 Songkittiguna, P. 55 Sparks, H.V. 56 Stephens, N.L. 57 Stoclet, J.C. 34,50,62 
Temnyahov, N. 58 Toda,N. 59 Trapani. A. 26,60,61 Travo,P. 62 Traystman, R.J. 63

Trevethick, M.A. 64 Turlapaty,P.DJVl.V. 65

Uvelius,B, 66

VanBeek,J. 67

Vanhoutte, P.M. 15, 16, 17, 27,

68,70,72 VanNueten,J,M. 67,68 Vatner, S.F. 69 Verbeuren, T.J. 70 Verhaeghe,R.H. 28

Vila,C. 2 Voelkel,N.F. 71

Wattus,G.D. 47 Webb,R.C. 72 Wester, A. 14 Willems, J.L. 9 Willemse, PJ. 14 Winn,H.R. 73

Winquist, R.J. 74 Worcel, M. 61,75 Wurm,A. 31

Yamamoto, Y. 76

Zanchetti, A. 36 Zawadski, J. 23 Zelis, R. 77 Zelyazkow, D. 58 